\title{
Building performance indicators and IEQ assessment procedure for the next generation of EPC-s
}

\author{
Karl-Villem Võsa ${ }^{1 *}$, Andrea Ferrantelli ${ }^{1}$, Dragomir Tzanev $^{2}$, Kamen Simeonov $^{2}$, Pablo Carnero ${ }^{3}$, Carlos Espigares $^{3}$, \\ Miriam Navarro Escudero ${ }^{3}$, Pedro Vicente Quiles ${ }^{4}$, Thibault Andrieu ${ }^{5}$, Florian Battezzati ${ }^{5}$, Katia Cordeiro ${ }^{5}$, Francis \\ Allard ${ }^{5}$, Zoltan Magyar ${ }^{6}$, Giusy Turturiello ${ }^{7}$, Luca Alberto Piteràn ${ }^{7}$, Simona d'Oca ${ }^{8}$, Eric Willems ${ }^{8}$, Peter Op 't Veld ${ }^{8}$, \\ Andrei Vladimir Lițiu ${ }^{9}$, Cătălin Lungu ${ }^{10}$, Tiberiu Catalina ${ }^{11}$ and Jarek Kurnitski ${ }^{12,1,11}$
}

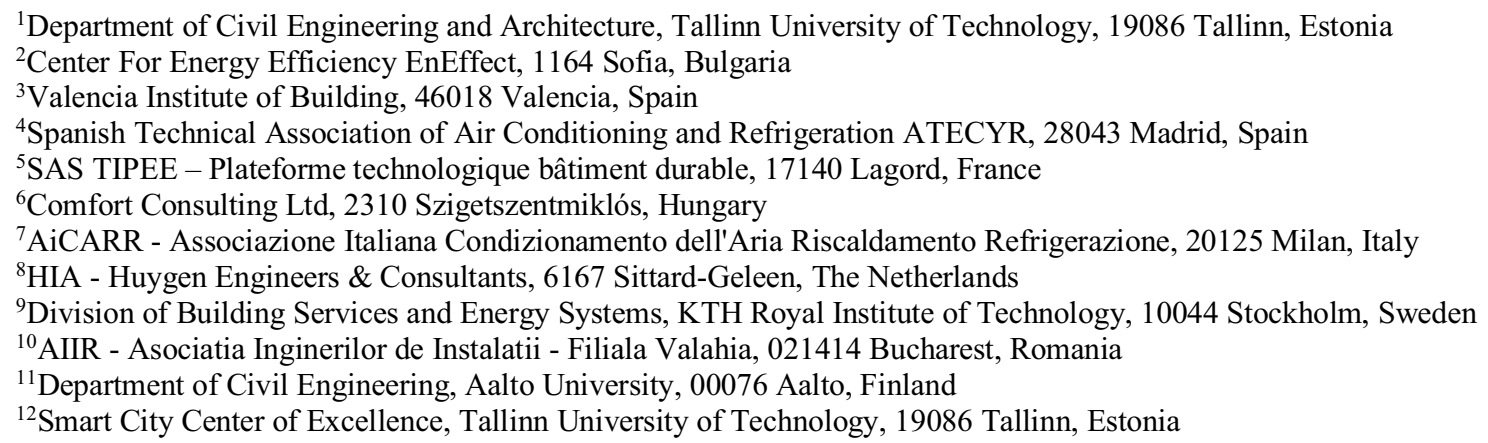

\begin{abstract}
In the current implementation of EPC-s, the assessment focus is purely on the energy consumption data. For the next generation of EPC-s, new performance indicators are proposed to address relevant building performance aspects, such as sustainability, productivity and market value. These indicators would enable evidence-based decision-making processes and facilitate the delivery of renovation triggers. Within the EPC framework, the problem is not the availability of such performance indicators, but the assessment effort required. Only easily available data can justifiably be introduced to bulk EPC-s, either as direct complementary input or as a performance indicator. Availability of such data was analysed from case studies that included EPC-s from $11 \mathrm{EU}$ member states, mainly non-residential buildings. Analysed data included relevant HVAC information such as ventilation air flows, heating and cooling set-points and installed power, but also output data, such as EPC classes, net and primary energy need and GHG emissions. Based on our findings, we outlined two different development paths - one for existing buildings and one for new buildings and major renovations. Two categories of complementary indicators to energy are proposed - IEQ and power indicators.
\end{abstract}

\section{Introduction}

The effects of exponential growth in resource consumption have been explored and contested for many decades. It is generally accepted that BREEAM (Building Research Establishment Environmental Assessment Method) is the world's first green building rating tool and was established in 1990 [1]. Many similar schemes have since been developed with varying numbers of assessed parameters and levels of detail, such as LEED and Green Star [2][3]. These are voluntary schemes that attempt to rate or reward relative levels of compliance or performance with environmental requirements and goals. These rating schemes assess the performance of buildings in categories such as building materials, energy efficiency, water efficiency, waste management, land use and ecology, management, pollution, IEQ (Indoor Environmental Quality), location, innovation in design, emissions, and many other categories. These schemes can be applied to different types of buildings, e.g. new and existing buildings, residential and non-residential buildings and address different criteria, e.g. energy performance only or wider sustainability schemes.

These voluntary schemes find varying levels of use in different EU member states. Reasons for limited implementation include high costs of international schemes, lack of market demand and unawareness of the advantages and value that the schemes offer. In member states with low uptake, the schemes are mainly used by international investors and developers that operate in multiple countries and regions where the certification is seen as lucrative.

\footnotetext{
* Corresponding author: karl-villem.vosa@taltech.ee
} 
These schemes co-exist with the mandatory Energy Performance Certificates (EPC-s) required by the EPBD [4]. EPC-s relate mostly to designed or measured energy performance of the building. The main indicator of the EPC scheme is the EPC class, but generally other indicative values such as net and/or primary energy consumption, system-by-system energy breakdown, $\mathrm{CO}_{2}$ emissions and delivered and exported energy values (share or renewable energy) are available. Consequently, the energy performance is well-described in the EPC-s, but sustainability and other performance indicators are not.

When considering indicators for the next generation of EPC-s, it is evident that the main problem does not lie with the availability of indicators, but in the assessment effort and whether the EPC issuer is qualified for such an assessment. Most voluntary certification rating schemes are mainly targeted towards non-residential property investors and developers, e.g. larger industrial and commercial buildings. On the other hand, EPC scheme involves all buildings regardless of their type and size. There is a considerable difference in the assessment effort of these two types of schemes, and for bulk EPC-s, the cost and the level of qualification needed for voluntary schemes cannot be matched. Consequently, only a selection of assessable parameters can be feasibly proposed as new performance indicators in the next generation of EPC-s.

An important set of proposed parameters relate to the IEQ (indoor environment quality) of the building. These parameters are room temperature (overall thermal comfort of the occupant), ventilation rates and $\mathrm{CO}_{2}$ levels (indoor air quality), draught rates (thermal discomfort) and HVAC system noise (acoustic comfort). The TAIL indicator developed in ALliance for Deep RENovation (ALDREN) is one example of a voluntary IEQ indicator, outlined are compatible parameters for inclusion as new indicators in EPC-s [5][6]. Another example is the Estonian Green Label, which also assesses the IEQ from a similar list of parameters, along with the energy and location category indicators based on the research concluded in [7]. These are both examples of very simple certificates, with only three or four levels of classification.

In this publication, we studied different EPC-s from 11 EU member states to establish which data is readily available and can be implemented for new indicators in the next generation of EPC-s. Based on our findings, we outline two different development paths - one for existing buildings and one for existing buildings. Complementary to the current EPC-s, power IEQ and power indicators are also proposed to be included.

\section{Methods}

Our research is based on a broad literature review on the currently available performance indicators. It could be concluded that there are many appropriate indicators readily available on the market already, with varying levels of uptake in the building sectors of different countries. For bulk application within the current EPC framework, they are not appropriate, mainly due to the relatively high time and monetary costs involved.

With this in mind, we decided to assess whether it is possible to expand on the data already available in the EPC-s for the development of improved performance indicators.

\subsection{Case studies}

A total of 11 EPC-s from different EU member states were included in the case study. These were mainly EPC-s for larger buildings, such as office and educational buildings, as seen in Table 1. Most of the assessed buildings have been fairly recently constructed or renovated, while only two were built or renovated before year 2000 .

Data availability from these EPC-s was analysed in eight distinct categories as shown in Table 2. Even from the subdivision it is evident that, there is more readily available data that could be used for further assessing the performance of a building. The data was then aggregated to represent availability of each data point among all the buildings in the case study.

\subsection{Proposal of IEQ assessment and power indicators}

From the available data, we establish that the indoor environment quality can potentially be assessed. A distinction is made between new and existing buildings regarding how this can be methodologically accomplished. Furthermore, we consider including power indicators based on either design documentation or measurement data. These are discussed in detail in sections 4 and 5 .

Table 1 - Case study building descriptions.

\begin{tabular}{|c|c|c|c|c|}
\hline 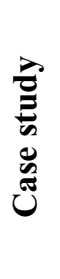 & 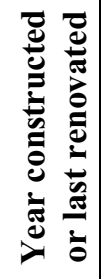 & & 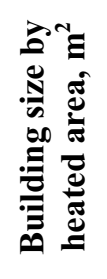 & 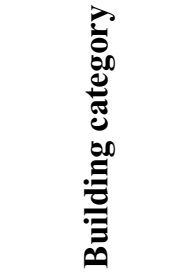 \\
\hline 1 & 2016 & Netherlands & 6267 & Nursing \\
\hline 2 & 2014 & Sweden & 21244 & Office \\
\hline 3 & 2018 & Estonia & 2170 & Office \\
\hline 4 & 2014 & Hungary & 2243 & Educational \\
\hline 5 & 2008 & Spain & 8520 & Office \\
\hline 6 & 2014 & Slovenia & 16256 & Educational \\
\hline 7 & 2017 & Romania & 8058 & Multi-family \\
\hline 8 & 1999 & Italy & 2972 & Educational \\
\hline 9 & 2010 & Bulgaria & 306 & Two-family \\
\hline 10 & 1997 & France & 9200 & Library \\
\hline 11 & 2009 & Denmark & 950 & Office \\
\hline
\end{tabular}


Table 2 - Parameters assessed in EPC data availability.

\begin{tabular}{|c|c|}
\hline Category & Assessed parameter \\
\hline \multirow{9}{*}{ Envelope } & Element-by element breakdown \\
\hline & Thermal transmittance \\
\hline & Element surface areas \\
\hline & Orientation of façade elements \\
\hline & Glazing solar heat gain coefficient \\
\hline & Total heat loss coefficient \\
\hline & Thermal bridges \\
\hline & Infiltration \\
\hline & Closure type \\
\hline \multirow{5}{*}{ Ventilation } & Type - Natural/mechanical \\
\hline & Annual energy consumption \\
\hline & Air flow rate \\
\hline & Specific fan power \\
\hline & Heat exchanger efficiency \\
\hline \multirow{6}{*}{$\begin{array}{l}\text { Heating } \\
\text { system }\end{array}$} & Any data available? Yes/no \\
\hline & Annual energy consumption \\
\hline & Generation type \\
\hline & Generation efficiency \\
\hline & Installed power \\
\hline & Emitter/distribution type \\
\hline \multirow{6}{*}{$\begin{array}{l}\text { Cooling } \\
\text { system }\end{array}$} & Any data available? Yes/no \\
\hline & Annual energy consumption \\
\hline & Generation type \\
\hline & Generation efficiency \\
\hline & Installed power \\
\hline & Emitter/distribution type \\
\hline \multirow{5}{*}{$\begin{array}{l}\text { DHW } \\
\text { system }\end{array}$} & Any data available? Yes/no \\
\hline & Annual energy consumption \\
\hline & Generation type \\
\hline & Generation efficiency \\
\hline & Accumulation tank capacity \\
\hline \multirow{3}{*}{ Lighting } & Any data available? Yes/no \\
\hline & Annual energy consumption \\
\hline & Installed power \\
\hline \multirow{5}{*}{ Renewables } & Any data available? Yes/no \\
\hline & Annual energy production \\
\hline & Generation type \\
\hline & Generation efficiency \\
\hline & Installed power \\
\hline \multirow{7}{*}{$\begin{array}{c}\text { Energy } \\
\text { performance }\end{array}$} & EPC class \\
\hline & Energy breakdown by systems \\
\hline & Net energy demand \\
\hline & Primary energy demand \\
\hline & GHG emission, $\mathrm{CO}_{2}$ equivalent \\
\hline & Energy breakdown by carrier \\
\hline & Partial indicators \\
\hline
\end{tabular}

\section{Results}

The data availability from the case study EPC-s are shown in Figure 1-Figure 8.

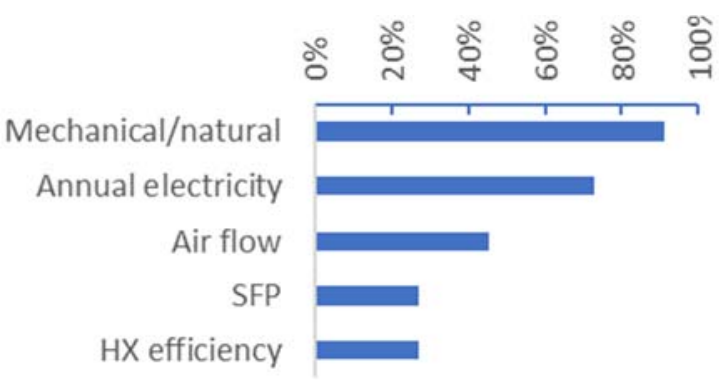

Figure 1 - Ventilation data availability

In almost all cases, the ventilation system was described in at least some detail. Surprisingly, only $45 \%$ of the EPC-s listed the rated air flows, with even smaller portion describing the fan performance or heat exchanger efficiencies. Relevant data that was also occasionally present:

- Zone supply temperature set-points

- Frost protection temperature of the heat exchanger

- Breakdown of CAV/VAV systems

- Description of the control strategy (user profiles, extract temperature, $\mathrm{CO}_{2}$ level, free cooling)

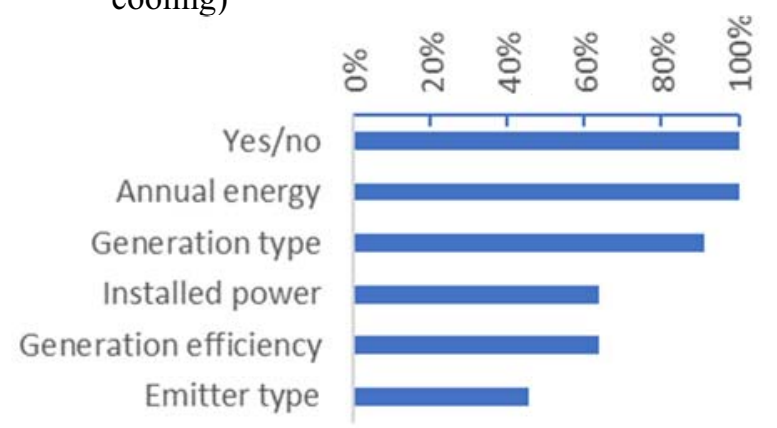

Figure 2 - Heating data availability

The heating system is well-described in all submitted EPC-s. Most EPC-s showed the installed power, heat generation type and efficiency. Emitter types were described in just over half the EPC-s. Relevant data that was occasionally present:

- Control strategy (thermostatic valves, room sensors)

- Distribution losses

- Circulation pump and insulation data 


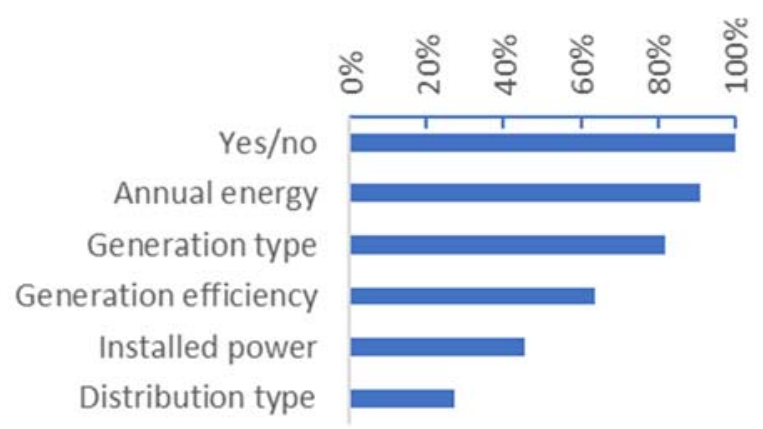

Figure 3 - Cooling data availability

The cooling system is also well-described in all studied buildings similarly to the heating system. Relevant data that was occasionally present:

- Control strategy - free cooling (GSHP, nighttime ventilation)

- Distribution losses

The same can be said for the DHW and lighting systems.

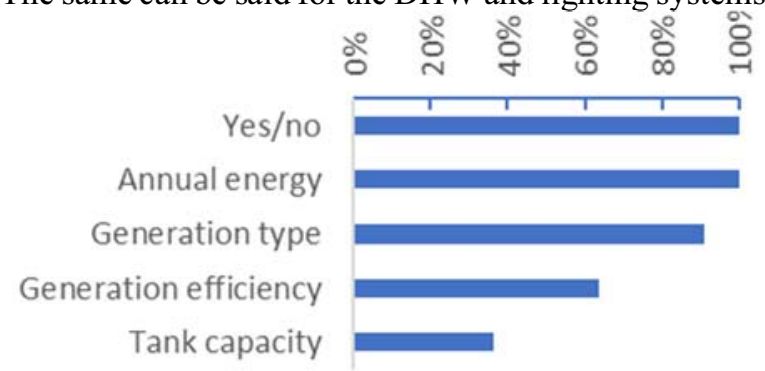

Figure 4 - DHW data availability

Relevant data that was occasionally present:

- Number of taps, shower, toilets etc

- Distribution losses

- Data about circulation pumps, pipe insulation

- Greywater heat recovery

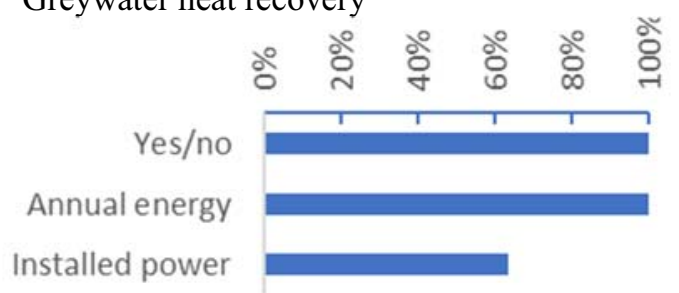

Figure 5 - Lighting data availability

Relevant data that was occasionally present:

- Illuminance (lx), efficiency $\left(\mathrm{W} /\left(\mathrm{m}^{2} \mathrm{~lx}\right)\right)$

- Breakdown of lamp types (LEDs, CFLs, incandescent)

- Control strategy (motion sensors, daylight sensors)

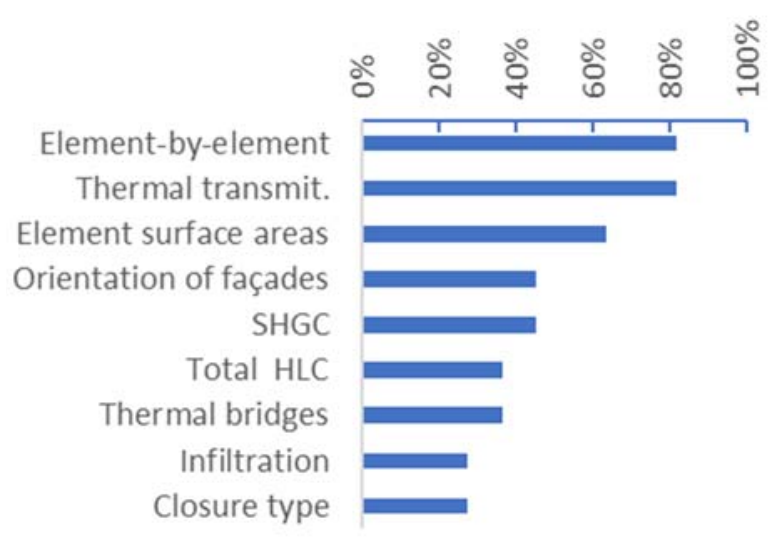

Figure 6 - Envelope data availability

Envelope data is generally well described, typically a break-down of thermal properties of all or typical envelope elements is given (walls, floor, roof, windows, doors and other openings). For glazed surfaces, generally the SHGC or another equivalent parameter was given. In a smaller portion of buildings, infiltration (airtightness) and thermal bridge data was also given. Relevant data that was occasionally present:

- building thermal capacity $(\mathrm{kJ} / \mathrm{K})$

- (hygro)thermal properties of individual material layers

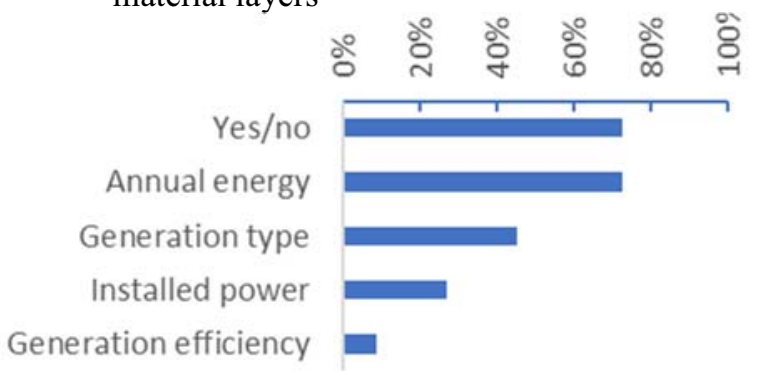

Figure 7 - Renewables data availability

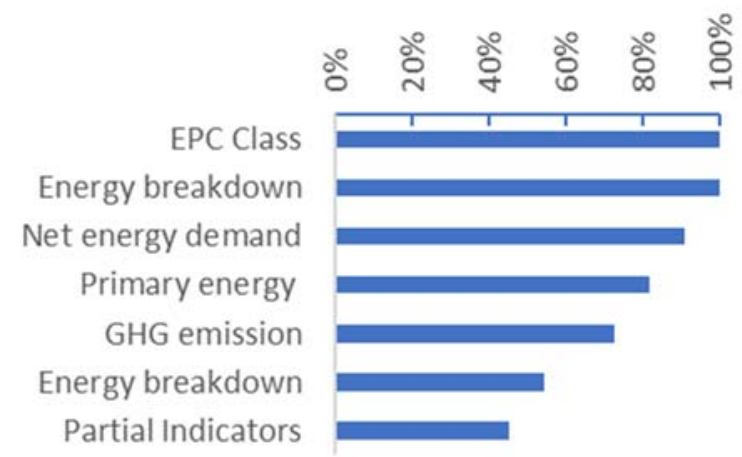

Figure 8 - EPC data availability

Presented energy data is almost perfect - along with the mandatory EPC class, also the energy breakdown by systems and net energy demand of the building is present in all but one of the case studies. In two cases, the primary energy indicators have not been established.

More significantly, there are no power indicators present in the current implementation of EPC-s. 


\section{IEQ assessment}

\subsection{Indoor climate category assessment in new buildings}

Design documentation of the building must state the design criteria of the indoor environment parameters. These parameters must include ventilation rates, heating and cooling set-point temperatures, maximum air velocities and HVAC noise in major space categories. Furthermore, calculation or simulation reports that prove that the design criteria are met must also be appended to the documentation where applicable. If this data is present, then there are no further actions needed to assess the indoor climate category. If this data is not present or is partially missing, then the relevant calculations or simulations must be conducted and submitted for the indoor climate category assessment.

\subsection{Indoor climate category assessment in existing buildings}

Generally, assessment of the building indoor climate category begins with checking compliance with indoor category III, then compliance with category II and finally with category I. If the building does not comply with category III, then it will belong to indoor climate category IV. Assessment of these categories requires different levels of knowledge and expertise from the assessor. For categories III and IV, on-site inspection and brief overview of design documents should provide enough information to make the assessment. This level of assessment could be done by people who have some knowledge of indoor climate and HVAC systems, for example construction engineers, building designers and heads of maintenance and administration. For correct assessment of categories I and II, expert knowledge of HVAC systems, indoor climate, building physics and measurement technology is required. The assessment process involves thorough on-site inspections, detailed review of design documentation, on-site measurements, simulations of indoor climate, and for category I, indoor climate questionnaires for occupants. Therefore, this level of assessment can be done only by qualified assessors. One example of a data collection template for these procedures can be found in [8].

\subsubsection{Process of indoor climate category assessment in existing buildings}

Prior to the on-site inspection, it is recommended to contact the building owner, administrator or the person responsible for maintenance to gather additional or missing information for the assessment process. In particular, the measurement protocol of the ventilation system is needed to cross-check design air flow rates and noise (sound pressure) levels. Additionally, as-built drawings of HVAC systems are needed to verify the integrity of the on-site systems. If the ventilation documentation is not provided, then air flow measurements must be carried out on-site.
It is also necessary to determine characteristic and critical rooms of the building where measurements will be carried out if necessary.

Characteristic rooms - most typical rooms within the building with constant occupancy, such as office, class or living rooms or individual workspaces in an open office setting. Generally, 3-10 rooms are required for a representative sample. It is necessary to consider the following:

- intended use of the rooms, e.g. office space, classroom, living room etc.

- orientation of the rooms with respect to the cardinal directions

- presence of HVAC systems, e.g. rooms with and without air conditioning devices

Critical rooms - rooms where thermal comfort problems are most likely to occur, such as rooms with large, glazed surfaces or high internal heat gains. Generally, 3-10 rooms are required for a representative sample. Examples of critical rooms include but are not limited to rooms where:

- air conditioning devices are missing, or selected devices are improper for the room type and load

- glazed surfaces constitute a considerable area of the building envelope

- windows are oriented to the southern and western facades (risk of overheating)

- windows are in different facades (corner rooms)

- glazed surfaces are not shaded

- internal gains are higher than typical

- air flow rates are lower than typical and where supply air is not cooled

- opening of windows is not possible or is limited

\subsubsection{Indoor climate category III and IV assessment}

Category III is achieved when the following criteria are fulfilled:

- Heating system is present and the heat output in rooms with regular occupancy can be controlled on a room or zone basis

- Mechanical ventilation is present in rooms with regular occupancy. Air flow rates or $\mathrm{CO}_{2}$ levels must be in accordance with EN 16798-1 [9]

- Rooms with regular occupancy where glazed surfaces are on the east, south-east, south, south-west or west facades have mechanical cooling, and the indoor temperature can be controlled on a room or zone basis. Alternatively, the absence of cooling devices can be justified with a simulation calculation in critical rooms

If a measurement protocol of the ventilation system is not provided, then the air flow rates in characteristic rooms must be measured and checked against reference values in EN 16798-1. Alternatively, $\mathrm{CO}_{2}$ levels can be measured and compared to EN 16798-1 during at least 4 hours of occupancy instead.

The measurement periods in a non-residential building must be at least four hours in typical use 


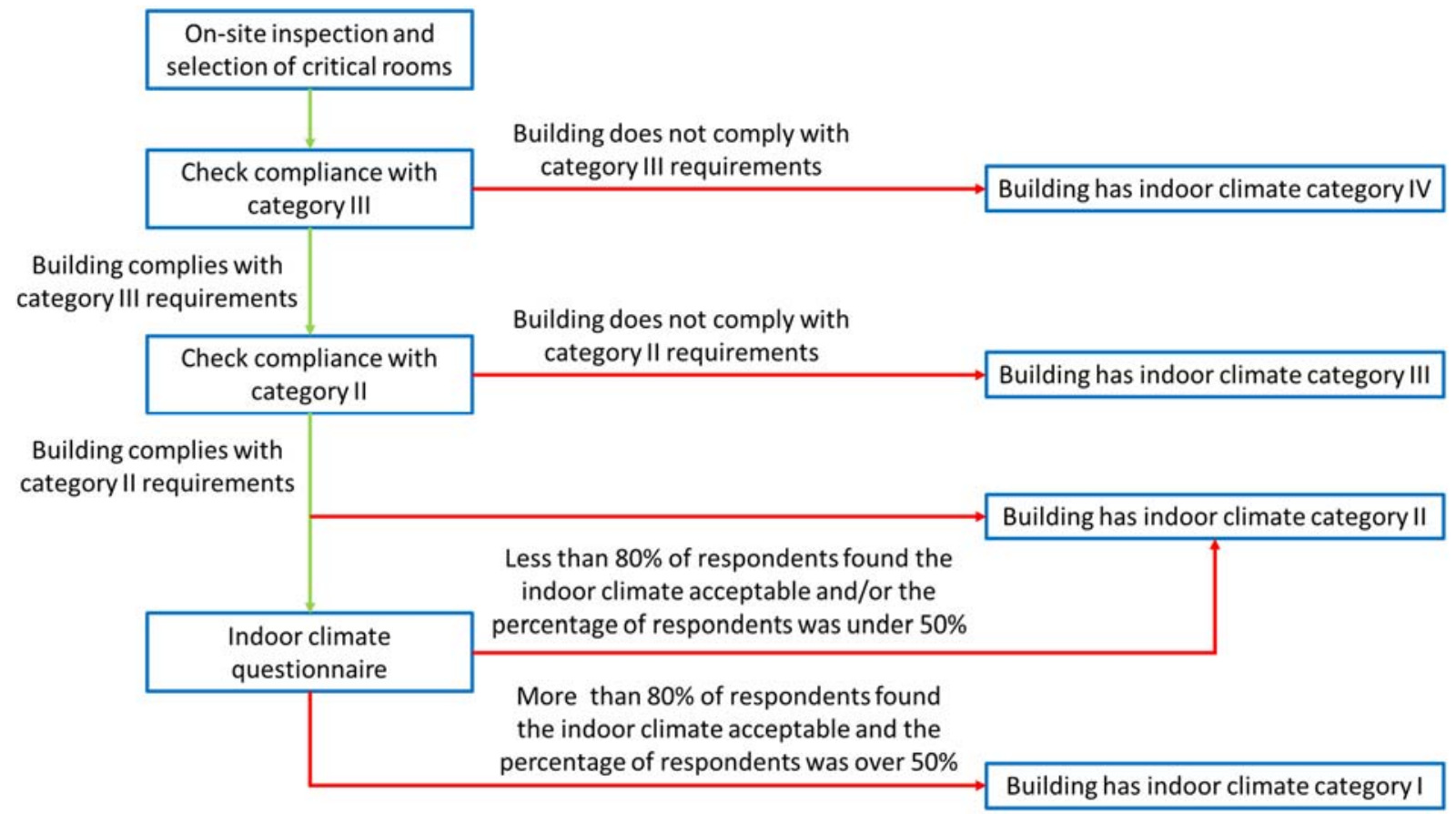

Figure 9 - Process of indoor climate category assessment

conditions. Air flow rates may alternatively be assessed by measuring each air handling unit's total air flow rate vs design conditions in served spaces, given that the device is working at the intended capacity and its air filters are clean.

\subsubsection{Indoor climate category I and II assessment}

Assessment of compliance to these levels requires simulation or measurement of indoor climate along with a thorough review and analysis of design and as-built documentation of HVAC systems. Both calculated and measurement-based assessments are allowed, as well as a combined approach for different technical systems

On-site inspections and review of documentation shall verify that:

- chosen technical solutions can maintain required levels of indoor climate parameters in all rooms with regular occupancy

- HVAC systems are in working condition and match with as-built drawings and documentation

- normative air flow rates and sound pressure levels have been considered in the system design and are demonstrated in measurement protocols.

For calculation-based approach, the following calculations or simulations are required:

- cooling load calculations for characteristic and critical rooms

- terminal and room unit air flow jet analysis in manufacturer or validated third party software for characteristic and critical rooms

For measurement-based approach, the following measurements are required:

- air temperature measurements during the heating and cooling season in characteristic and critical rooms. Minimum length of measurements shall be at least one month and must include periods with typical heating and cooling loads.

- air velocity measurements during the cooling season with the cooling device operating and during the cooling season without cooling load

- air flow rate and sound pressure level measurements if measurement protocols are not available.

For category I assessment, indoor climate survey must be done among the occupants in addition to the previously described step. The questionnaire must cover the following aspects:

- room air temperature (hot, warm, slightly warm, neutral, slightly cool, cool, cold)

- $\quad$ air quality (according to EN 16798-2 [10])

The whole process is outlined in Figure 9.

\section{Discussion}

The review of existing performance indicators shows that in the context of EPC development, the main problem is not the availability of indicators, but rather the required assessment effort and qualification of the EPC issuer. A plethora of voluntary schemes provide IEQ, energy performance, power and many other indicators, but generally the amount of necessary input data, time and competence of the assessor exceed the levels established under the current implementation of EPC-s especially for existing buildings.

\subsection{New buildings and major renovations}

For the inclusion of new generation performance indicators, EPC-s should be based on dynamic simulations or hourly calculations for energy and room 
temperatures, otherwise the IEQ performance and power assessment would not be possible to conduct or additional simulations, basically with the extent of another EPC would be needed. However, in many countries, EPC-s for new buildings are already simulated with commercial dynamic simulation tools or with simplified hourly tools, in which cases an involvement of new performance indicators could be seen as natural development step.

\subsubsection{IEQ performance indicators}

IEQ indicators are generally divided into 3-4 categories, such as thermal comfort, air quality, acoustics, visual comfort etc. These indicators are mainly applicable for new buildings - design documentation should include the IEQ criteria to which the building was designed along with relevant calculation or simulation results and measurements where necessary.

Some of the data required for IEQ assessment is already present in EPC-s of selected countries. Such data are ventilation rates, heating and cooling setpoints and cooling system data. Based on this existing data, general thermal comfort, air quality and noise categories can be easily determined while local thermal discomfort assessment would be typically needed to determine the category of the thermal comfort in the comprehensive and reliable fashion.

Complete IEQ assessment requires typically some additional design tasks and competence of the designer/assessor. These additional design tasks should collect information about air distribution to assess air velocities, i.e. to conduct supply air devices jet calculation with relevant software in representative rooms that is very important for occupant comfort and wellbeing because draught complaints are one of the most common complaints in modern offices. In some cases, an additional room temperature and cooling load simulations may also be required, but in many cases, these are already included in the design of HVAC \& energy.

\subsubsection{Power indicators}

Current EPC-s consider only the energy expenditure of the building, while the power needs are completely neglected. Existing energy performance minimum requirements may lead to situation where buildings with similar energy expenditures, but different power profiles may cause highly different load for the corresponding distribution grids (electricity, district heating, district cooling) in a contrasting manner.

Furthermore, energy flexibility is an issue raised by Smart Readiness Indicator, but is currently not addressed in any EPC:

- Responding to the grid demand and supply allows for (peak) power shifting, as well as using alternative means of energy production or storage, e.g. renewables if available on-site.
- It is expected that some flexibility indicators could be developed under the Smart Readiness Indicator assessment, where grid flexibility and demand-response are evaluated parameters

Starting point for flexibility indicators can be simple delivered and exported energy duration curves with hourly data by energy carrier which help to describe the effect of the building on distribution grids:

- More flexibility indicators may be developed within the Smart Readiness Indicator framework

- Simple and robust power indicator is to consider the $\left(5^{\text {th }} / 95^{\text {th }}\right.$ percentile of hourly peak power loads $\mathrm{W} / \mathrm{m}^{2}$ ) of delivered and exported energy.

\subsection{Existing buildings}

Often very limited data is used (and in many cases available) when EPC-s for existing buildings are prepared. This can lead to a very crude estimation of the energy performance of the building, with the focus on keeping the issuing cost of the EPC low rather than providing an accurate actual energy use or energy performance. As rather a common practice, default values are often assumed, and available design documentation is not inspected and used in its full detail, and in some cases EPC-s may not be based or include measured energy data.

Detailed inspection and measurements to conduct more comprehensive assessment of energy and IEQ can be seen completely unrealistic in current schemes and practices for existing buildings, because:

- These procedures are usually very timeconsuming, including data collection and measurements which are typically not available when compared to current implementation of measured energy used based EPC-s in existing buildings. This increase in workhours more by factor 10 of the assessor will raises the cost of the EPC above feasible levels.

- EPC assessors do not necessarily possess the correct skillset or competence to accurately conduct such inspections and measurements.

- To be meaningful and accurate, one needs to conduct indoor climate surveys along with measurements - such a service is targeted to interested clients/owners, but not suitable for bulk EPC-s.

While IEQ assessment in existing buildings is complicated, power indicators can be easily implemented in existing buildings as well, under the assumption that hourly metering data is available, or such smart meters would be installed. 


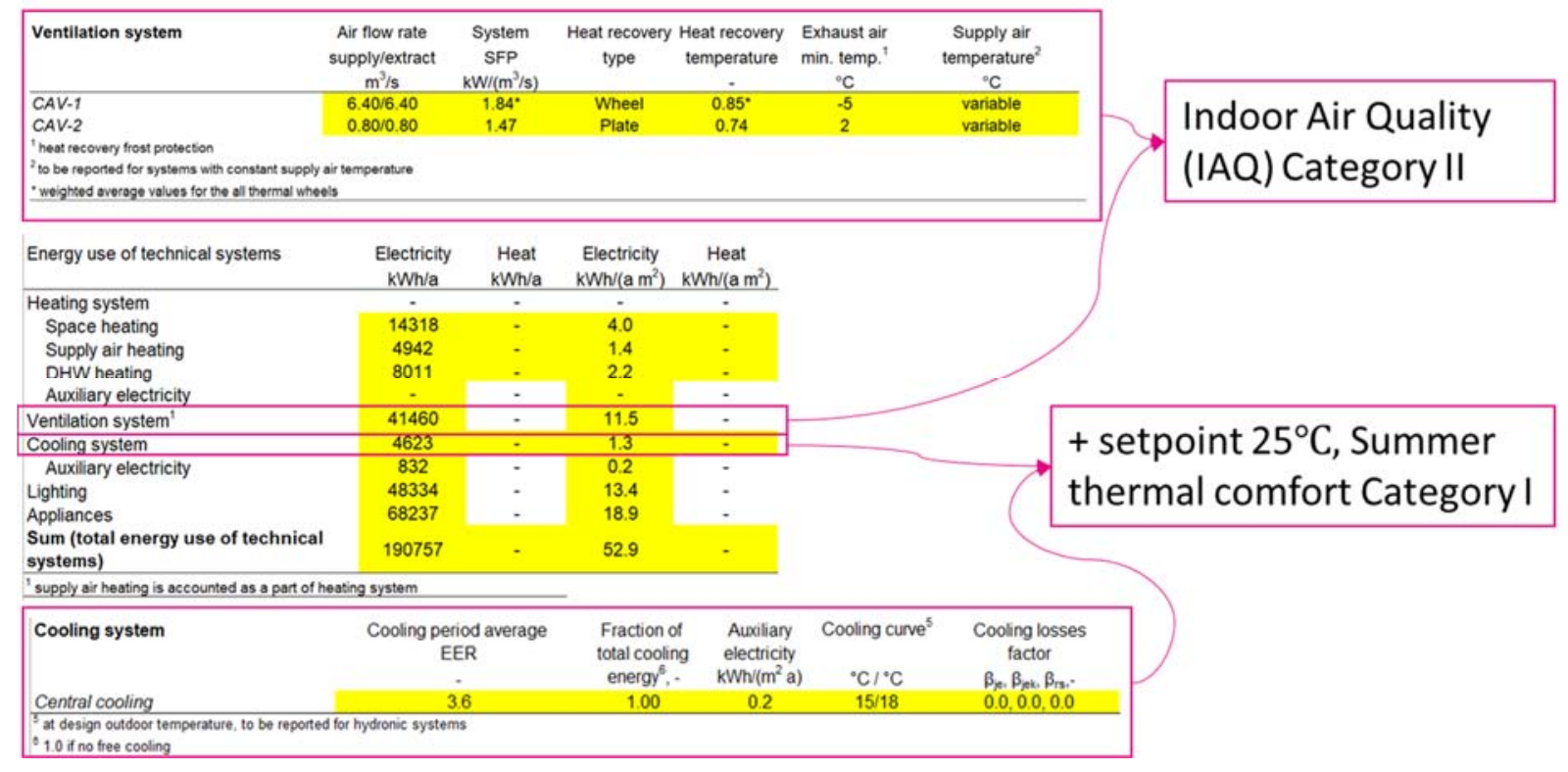

Figure 10 - An example of already available data in existing Estonian EPC which allows to conduct partial IEQ assessment of a new building, showing IAQ Category II and summer general thermal comfort Category I

Measured energy usage and energy cost data could be included to increase the credibility of EPC-s for end customers. Running energy expenditure and costs in Euros are a lot more tangible to the end user than tier/category-based indicators, which are generally perceived as relative performance indicators (e.g. category $\mathrm{A}$ is better than category $\mathrm{B}$, but by how much is often misunderstood or disregarded). Total energy expenditure (electricity/heat/other) of the building is generally metered and available even if sub-metering data by systems is not.

\section{Conclusions}

In the current implementation of EPC-s, the assessment focus is purely on the energy consumption data of buildings. Furthermore, for existing buildings, they are often made at a low cost and not in alignment with actual energy consumption of the building. For new buildings and major renovations, the EPC-s are generally calculated during the design process of the building when all design documentation is available but is utilized only for the aspects minimally required by the EPBD. In this article we outlined performance indicators that could be implemented in the framework of next generation EPC-s based on a case study of 11 EPC-s. Within the EPC framework, the problem is not the availability of performance indicators, but with the assessment effort required (cost and level of qualification necessary) for these indicators. There are plenty of privately developed voluntary certification schemes readily available both locally and internationally, such as LEED, BREEAM and Green Star, but these are typically targeted at interested investors and developers and are not suitable for bulk EPC-s. Only the data that is easily available can justifiably introduced to EPC-s either as direct complementary input or as a performance indicator. We propose two different development paths - one for existing buildings and one for new buildings and major renovations. Two categories of complementary indicators to energy are proposed - IEQ and Power indicators.

U-CERT Consortium would like to acknowledge the financial support of the European Commission under the H2020 programme. This project has received funding from the European Union's Horizon 2020 research and innovation programme under grant agreement number 839937.

\section{References}

[1] BREEAM, "Technical Manual (2016), BREEAM International New Construction," Build. Res. Establ. Gr., 2016.

[2] U.S. Green Building Council, "LEED v4 User Guide," United State Green Building Council. 2014.

[3] GBCA, "What is Green Star?| Green Building Council of Australia," Green Building Council of Australia, 2020. .

[4] European Parliament, "Directive 2002/91/EC of the European Parliament and of the Council of 19 May 2010 on the energy performance of buildings (recast)," Off. J. Eur. Union, vol. L 153/13, 2010.

[5] M. M. Sesana, G. Salvalai, D. Brutti, C. Mandin, and W. Wei, "ALDREN: A methodological framework to support decisionmaking and investments in deep energy renovation of non-residential buildings," Buildings, vol. 11, no. 1, 2021, doi: 10.3390/buildings 11010003 .

[6] P. Wargocki, C. Mandin, and W. Wei, "Aldren-Tail index for rating IEQ," ASHRAE J., vol. 61, no. 12, 2019. 
[7] E. Seinre, J. Kurnitski, and H. Voll, "Building sustainability objective assessment in Estonian context and a comparative evaluation with LEED and BREEAM," Build. Environ., vol. 82, pp. 110-120, Dec. 2014, doi: 10.1016/j.buildenv.2014.08.005.

[8] K. Ahmed and J. Kurnitski, "NERO - D1.1 Data collection templates and inspection procedures." 2017.

[9] European standards committee, "prEN 167981:2015 Indoor Environmental Input Parameters for Design and Assessment of Energy Performance of Buildings Addressing Indoor Air Quality, Thermal Environment, Lighting and Acoustics," 2015.

[10] CEN/TR 16798, “CEN/TR 16798-2:2019

Energy performance of buildings - Ventilation for buildings - Part 2: Interpretation of the requirements in EN 16798-1 - Indoor environmental input parameters for design and assessment of energy performance of buildings addressing Indo.” p. Module M1-6, 2019. 